\title{
Mortalidad de vacas en tres rebaños lecheros: estudio preliminar (1994-2004)
}

\author{
Cow mortality in three dairy herds: preliminary study (1994-2004) \\ L Rogel, R Tamayo* \\ Instituto de Medicina Preventiva Veterinaria, Facultad de Ciencias Veterinarias, Universidad Austral de Chile.
}

\section{SUMMARY}

The objective of this study was to determine the average annual mortality rate of dairy cows in three dairy herds of the Valdivia province, Chile, from 1994 to 2004; in addition the most common causes of death among the cows were determined. The information was gathered from 205 individual record cards of dead cows and related annual inventories, and analysed using Excel. The average gross mortality rate for the three farms was $4.8 \%$ whereas the rate of annual gross mortality for farm A was $6.8 \%$, farm B was $4.2 \%$ and farm C was $3.2 \%$. The most frequent causes of death when considering the three farms together were calving problems (21\%), undetermined causes (16.1\%) and digestive disorders (15.6\%). The highest mortality percentages were reached between April and September. Most of the cows died when they were seven years old (17\%), with the first stage of the lactation period showing the highest porcentage of dead cows $(61.8 \%)$. It was concluded that there is a high mortality rate in dairy farms and that the causes of death were different depending on the farm, but in general they were related to calving, undetermined causes, and digestive disorders.

Palabras clave: mortalidad, muertes, vacas lecheras.

Key words: mortality rate, death, dairy cattle.

\section{INTRODUCCION}

La muerte prematura de los animales es el resultado de la enfermedad, como también de accidentes o de una acción intencionada por terceros, y tiene un efecto pronunciado sobre la productividad de las poblaciones animales. En la ganadería intensiva, los costos de mortalidad son mayores cuando los animales con un alto potencial genético mueren durante los años de máxima producción (Wayne y col 1997).

La mortalidad entre vacas de lechería constituye un problema tanto en términos de pérdidas económicas (valor de las vacas muertas, disminución de la producción y mayor trabajo para los encargados de las vacas) como también al comprometer el bienestar del animal (sufrimiento antes de la muerte o eutanasia). Una evaluación de la mortalidad entre un grupo de vacas puede indicar diferentes niveles de salud y bienestar. La mortalidad puede estar influenciada por costos de reemplazo de las vacas, costos de sacrificio, gastos veterinarios, prácticas agrícolas y manejo (Thomsen y col 2004).

Desde un punto de vista económico, la mortalidad constituye una pérdida al contabilizar el valor individual de las vacas, en particular de las buenas productoras y del carácter irreversible del acontecimiento que implica un déficit genético irremediable y una suspensión de toda

Aceptado: 14.11.2006

* Casilla 567, Valdivia, Chile; rtamayo@uach.cl producción (leche, ternero) sin valorización post mortem (Faye y Pérochon 1995).

El patrón de mortalidad en rebaños lecheros organizados sirve como un indicador útil para evaluar el estado de salud del ganado y la eficacia de los programas de manejo. La disminución de los índices de morbilidad y mortalidad son los principales objetivos de manejo dentro del rebaño lechero (Prasad y col 2004).

Las estrategias para la prevención y control de enfermedades son componentes importantes de cualquier sistema de producción. Una estimación exacta del número de muertes y una evaluación de la importancia relativa de varios síndromes de enfermedades es de valor en la promoción de la salud del ganado, en la planificación de campañas de prevención de enfermedades y en la determinación de los temas que requieren de investigación adicional (Menzies y col 1995). Por lo tanto, la identificación correcta de la causa de muerte permite la aplicación adecuada de medidas que permitan prevenir pérdidas futuras (Miles y col 1998).

Un ambiente competitivo obliga al ganadero constantemente a adoptar nuevos y más intensivos métodos de producción con el objetivo de bajar los costos e incrementar la producción. La deliberación es si esta intensificación de la producción tiene un impacto adverso sobre la salud y el bienestar animal, con posibles efectos negativos en la calidad de vida de la población de vacas lecheras. Además, cambios en el ambiente fisiológico debido al incremento de la mecanización y el gran tamaño de los rebaños contribuyen a una pérdida de atención 
por vaca y a un aumento de la mortalidad (Norgaard y col 1999).

En el país son escasos los estudios sobre mortalidad de vacas lecheras, esto obedece a la falta de registros y de la información relacionada con el evento; además esta información tiene, en muchos casos, un carácter confidencial.

$\mathrm{Al}$ analizar varios estudios realizados en diferentes países, se obtuvo una tasa de mortalidad promedio de 2,3\% anual, con valores que fluctuaron entre un 1,0\% (Faye y Pérochon 1995) y un 4,6\% (Enyedi 1991). Las principales causas de muerte en estos estudios correspondieron en primer lugar a mastitis con un promedio de $13,5 \%$, con porcentajes que fluctuaron entre un $2 \%$ en Australia (Stevenson y Lean 1998) y un 22,6\% en México (Márquez y col 2001), junto con el grupo de causas desconocidas con un promedio de $27,6 \%$, con porcentajes que fluctuaron entre $14 \%$ en Dinamarca (Thomsen y col 2004) y un $46 \%$ en Inglaterra (Esslemont y Kossaibati 1997).

El período cercano al parto, incluido el inicio de la lactancia, es el período en que las vacas son más susceptibles a los desórdenes reproductivos y metabólicos que pueden conducir a la muerte (Markusfeld 1987, Stevenson y Lean 1998). Se puede resumir que el periparto o período de transición (3 semanas antes y 3 semanas después del parto) es un período críticamente importante para la salud, producción y rentabilidad de las vacas lecheras; y la mayor parte de los trastornos de salud ocurren durante este tiempo (Drackley 1999).

Los objetivos del trabajo fueron determinar la tasa de mortalidad por rebaño y para el período en estudio, las causas más frecuentes de muerte, los meses en que se producen los mayores porcentajes de muertes, frecuencia de distribución de las edades y la etapa de la lactancia de las vacas muertas.

\section{MATERIAL Y METODOS}

Para la realización del trabajo se utilizaron 205 tarjetas de registros individuales de las vacas muertas raza Frisón Negro de tres rebaños lecheros de la provincia de Valdivia (rebaños denominados A, B y C) desde los años 1994 al 2004 inclusive, además de 11 inventarios anuales correspondientes a los años en estudio.

De los inventarios se obtuvo, además del número de vacas al inicio de cada período y el número de vacas muertas por año, el número (registro predial) de cada vaca muerta en cada período, para después ser ubicado en las tarjetas individuales, las que habían sido ordenadas por año previamente, procediendo a obtener de cada tarjeta los siguientes datos: fecha de nacimiento, fecha del último parto, fecha de muerte y causa de muerte.

Cálculo de la tasa de mortalidad. La tasa de mortalidad fue calculada con la siguiente fórmula (Thrusfield 1990, Wayne y col 1997):

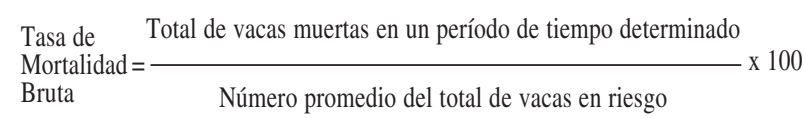

Origen de la causa de muerte. Las causas de muerte fueron registradas en su mayoría por los encargados de los rebaños (rebaño A: 72,4\% de las causas fueron registradas por los encargados y un $27,6 \%$ por el médico veterinario), ya que la periodicidad de las visitas realizadas por el médico veterinario (dos veces al mes para el rebaño A y una vez al mes para el rebaño B y C) rara vez permite estar presente en el momento de la muerte de las vacas ni realizar, por lo tanto, necropsias. Por ende, las causas de muerte registradas se basan en su mayoría en sintomatología ante mortem, existiendo escasos diagnósticos post mortem.

Sin embargo, para los rebaños B y C existe un mayor número de visitas adicionales del médico veterinario cuando se solicita su presencia por la existencia de animales enfermos, por el hecho de estar ubicados más cerca de la ciudad. El médico veterinario determina la patología que afecta al animal y prescribe un tratamiento, si se produce la muerte del animal el encargado registra el estado patológico señalado por el veterinario con anterioridad.

Clasificación de las causas de muerte. Debido a la gran cantidad de diagnósticos y signos descritos en las tarjetas individuales que señalan o identifican las causas de muerte de las vacas, se decidió clasificarlas en 8 causales de mortalidades, siguiendo el criterio de Thomsen y col (2004):

Trastornos derivados del parto: intoxicación puerperal, metritis séptica, muerte postaborto, muerte post cesárea, parto distócico, postradas posparto, prolapso uterino y ruptura uterina.

Trastornos metabólicos/nutricionales: hipomagnesemia, hipocalcemia y mal estado general (enflaquecimiento).

Trastornos digestivos: acidosis, actinobacilosis, cuerpo extraño, diarrea crónica, impactación ruminal, indigestión ruminal, intoxicación alimentaria, obstrucción intestinal, parasitismo severo, peritonitis, problema hepático, salmonelosis y timpanismo.

Trastornos podales: corresponden a problemas podales sin un diagnóstico exacto.

Mastitis: mastitis, mastitis crónica, mastitis por Escherichia coli y mastitis gangrenosa.

Accidentes: de diferente naturaleza, tales como, atropello en carretera, ahogo por caída a canales, fracturas y desgarros musculares, desagüe o pantanos y daños traumáticos.

Otros: edad, ruptura de recto, muerte durante tratamiento, muerte súbita, paro cardiaco, postradas, problema nervioso, problema respiratorio, robadas, muertas, y sacrificadas. 
No determinada: corresponde a vacas muertas encontradas por encargados de los rebaños sin ningún signo clínico previo de enfermedad.

Factores de variación. Se contemplaron una serie de factores de variación de la mortalidad de las vacas, como época del año, edad y etapa de la lactancia, por lo tanto, se determinó:

Porcentaje de mortalidad según meses del año: Se consideró el número de vacas muertas mensualmente durante todo el período en estudio, dividido por el total de vacas muertas durante el estudio y multiplicado por cien.

Porcentaje de mortalidad según edad: Se determinó la edad como los años de diferencia entre la fecha de nacimiento y la fecha de muerte de las vacas. Luego se calculó el porcentaje considerando el número de vacas muertas según su edad dividido por el total de vacas muertas durante el estudio y multiplicado por cien.

Porcentaje de mortalidad según etapa de la lactancia: Para calcular los días de lactancia que llevaban las vacas muertas se consideraron los días de diferencia entre la fecha del último parto y la fecha de muerte. Para evaluar la variación de los porcentajes de mortalidad en función de la etapa de la lactancia, ésta fue dividida en tres períodos:

Primer tercio (0 a 100 días)

Segundo tercio (101 a 200 días)

Tercer tercio (201 a 305 días)

Además, se agrupó a todas las vacas con más de 305 días de lactancia como de "cambio de temporada", por lo que este grupo no fue considerado en el cálculo de porcentajes de mortalidad según etapa de la lactancia. El porcentaje de mortalidad según etapa de la lactancia se calculó dividiendo el número de vacas según la etapa de la lactancia en que murieron por el número total de vacas muertas durante el período en estudio.

Todos los datos fueron ordenados y procesados en el programa computacional Microsoft Office Excel 2003 y se presentan como tablas y gráficos.

\section{RESULTADOS Y DISCUSION}

Tasas de mortalidad bruta anual de vacas lecheras de tres rebaños del sur de Chile, 1994-2004. Para describir el impacto cuantitativo de la muerte en la población de los rebaños se calcularon las diferentes tasas de mortalidad para los 11 años de estudio (cuadro 1), observándose en los tres rebaños una variación anual, con un promedio de $6,8 \%, 4,2 \%$ y $3,2 \%$ para las rebaños A, B y C, respectivamente. La alta tasa de mortalidad del rebaño A con respecto a los demás predios se puede explicar por el mayor tamaño de la población (A, B y C con 184, 76 y 82 vacas/año promedio) y, por ende, el mayor número de vacas en riesgo existentes en este rebaño.
Cuadro 1. Tasa de mortalidad bruta anual de vacas lecheras de tres rebaños del sur de Chile.

Annual gross mortality rate of dairy cows from three herds of southern Chile.

\begin{tabular}{lcccc}
\hline & \multicolumn{2}{c}{ Tasas de mortalidad (\%) } & \\
\cline { 2 - 3 } Años & Rebaño A & Rebaño B & Rebaño C & \\
& 2,8 & 0,0 & 2,6 & 1,8 \\
1994 & 5,2 & 4,4 & 2,7 & 4,1 \\
1995 & 6,6 & 1,3 & 4,2 & 4,0 \\
1996 & 6,0 & 5,2 & 1,4 & 4,2 \\
1997 & 9,1 & 6,8 & 0,0 & 5,3 \\
1998 & 10,2 & 3,9 & 5,0 & 6,4 \\
1999 & 6,8 & 10,1 & 5,1 & 7,3 \\
2000 & 6,0 & 2,6 & 2,4 & 3,7 \\
2001 & 7,1 & 6,2 & 4,0 & 5,8 \\
2002 & 9,2 & 4,8 & 2,9 & 5,6 \\
2003 & 5,8 & 1,3 & 5,4 & 4,1 \\
2004 & 6,8 & 4,2 & 3,2 & 4,8 \\
\hline \multirow{2}{*}{ Promedio } & \multicolumn{3}{c}{4,2} & \\
\hline
\end{tabular}

La tasa de mortalidad bruta promedio para todo el período en estudio considerando los tres rebaños fue de $4,8 \%$, valor mayor a lo reportado por otros países (Estados Unidos, Hungría, Colombia, Irlanda del Norte, Francia, Inglaterra, Australia), donde los rangos fluctuaron entre $0,96 \%$ y un $4,6 \%$ con un promedio de $2,3 \%$ (Gardner y col 1990, Enyedi 1991, Gómez 1992, Faye y Pérochon 1995, Menzies y col 1995, Esslemont y Kossaibati 1997, Stevenson y Lean 1998).

Las tasas de mortalidad en el rebaño A fluctuaron entre $2,8 \%$ y $10,2 \%$; en el rebaño B entre $0 \%$ y $10,1 \%$ y en el rebaño $\mathrm{C}$ entre $0 \%$ y 5,4\%. El año con mayor mortalidad considerando los tres rebaños fue el 2000 con un $7,3 \%$ promedio y el año con menor mortalidad fue en 1994 con un $1,8 \%$.

Causas más frecuentes de muerte en vacas lecheras desde el año 1994 al 2004. El número total de vacas muertas considerando los tres rebaños fue de 205 animales durante los años 1994 al 2004. Las causas de muerte más frecuentes se presentan en el cuadro 2.

$\mathrm{Al}$ considerar las causas de muerte de todas las vacas de los tres predios, se obtiene que las principales causales de mortalidad fueron los trastornos derivados del parto con un $21 \%$, las causas no determinadas con un $16,1 \%$ y los trastornos digestivos con un $15,6 \%$. Al comparar estos resultados con diferentes estudios (Argentina, Australia, Dinamarca, Estados Unidos, Francia, Inglaterra, Irlanda del Norte, México), en cuatro de ellos se menciona a los trastornos derivados del parto como la principal causa de muerte, con cifras que fluctuaron entre un 6\% (Stevenson y Lean 1998) y un 37,3\% (Márquez y col 2001). Al promediar todos los valores señalados en los cuatro estudios se obtuvo un $23 \%$, porcentaje similar al encontrado en este estudio. 
Cuadro 2. Causas más frecuentes de muerte de vacas lecheras en tres rebaños del sur de Chile desde el año 1994 al 2004 $(n=205)$.

Most frequent causes of death of dairy cows in three herds of the southern of Chile from 1994 to $2004(n=205)$.

\begin{tabular}{lr}
\hline Causas de muerte & $\begin{array}{c}\text { Total } \\
\%\end{array}$ \\
\hline Trastornos derivados del parto & 21,0 \\
No determinadas & 16,1 \\
Trastornos digestivos & 15,6 \\
Otros & 14,6 \\
Trastornos metabólicos/nutricionales & 13,7 \\
Accidente & 10,2 \\
Trastornos podales & 6,8 \\
Mastitis & 2,0 \\
\hline Total & 100,0
\end{tabular}

En relación a las vacas muertas sin una causa determinada, este grupo correspondió a vacas que no presentaron ningún signo clínico de enfermedad antes de morir y simplemente eran encontradas muertas por los encargados de los rebaños quedando registrada su causa de muerte como sin signos o desconocida, ya que no siempre se contaba con la disponibilidad del profesional para realizar la necropsia en forma oportuna. Esslemont y Kossaibati (1997) indican que el hecho que ninguna causa haya sido registrada para casi la mitad de las vacas muertas en su estudio sea probablemente porque la causa nunca fue establecida y porque pocos ganaderos examinan a las vacas post mortem. Este grupo es señalado por varios autores con porcentajes que fluctuaron entre un 14\% (Thomsen y col 2004) y un 46\% (Esslemont y Kossaibati 1997).
El porcentaje de vacas muertas por trastornos digestivos $(15,6 \%)$ es mayor que el descrito en la literatura por diversos autores (Gardner y col 1990, Faye y Pérochon 1995, Menzies y col 1995, Costa y col 2004 con un 9,9\%, $4,1 \%, 13,3 \%$ y $12,15 \%$, respectivamente).

Porcentajes de mortalidad según meses del año. Al analizar mensualmente la distribución de las muertes considerando los tres predios, éstas se produjeron principalmente desde abril hasta septiembre (figura 1), observándose el más alto porcentaje en el mes de agosto $(15,1 \%)$ y los más bajos en los meses de enero, febrero y marzo $(4,4 \%)$.

La principal causa de muerte de las vacas en los meses de mayor mortalidad, para abril $(47,6 \%)$, mayo $(21,1 \%)$ y agosto $(29 \%)$ fueron los trastornos derivados del parto; para junio $(26,3 \%)$ y septiembre $(25 \%)$ "otras causas", mientras que en julio los trastornos digestivos y los accidentes ambos con un 26,3\% fueron las causas que predominaron. Esto coincide con un estudio que indica que el patrón estacional de la mortalidad de las vacas tiende a relacionarse principalmente con los meses en los cuales se produce la mayor parte de los nacimientos, señalando que la mayoría de las muertes $(59,4 \%)$ de las vacas lecheras ocurre en el período de enero a mayo (período correspondiente a meses de invierno y primavera en el hemisferio norte) (Menzies y col 1995).

Porcentajes de mortalidad según edad de las vacas. En la figura 2 , se observa que la moda de la edad de muerte fueron los 7 años con un $17 \%$, esto considerando las vacas de los tres rebaños, observándose un bajo porcentaje de vacas muertas con once, doce y trece años, lo que es explicado por el bajo número de animales de esas edades en los rebaños.

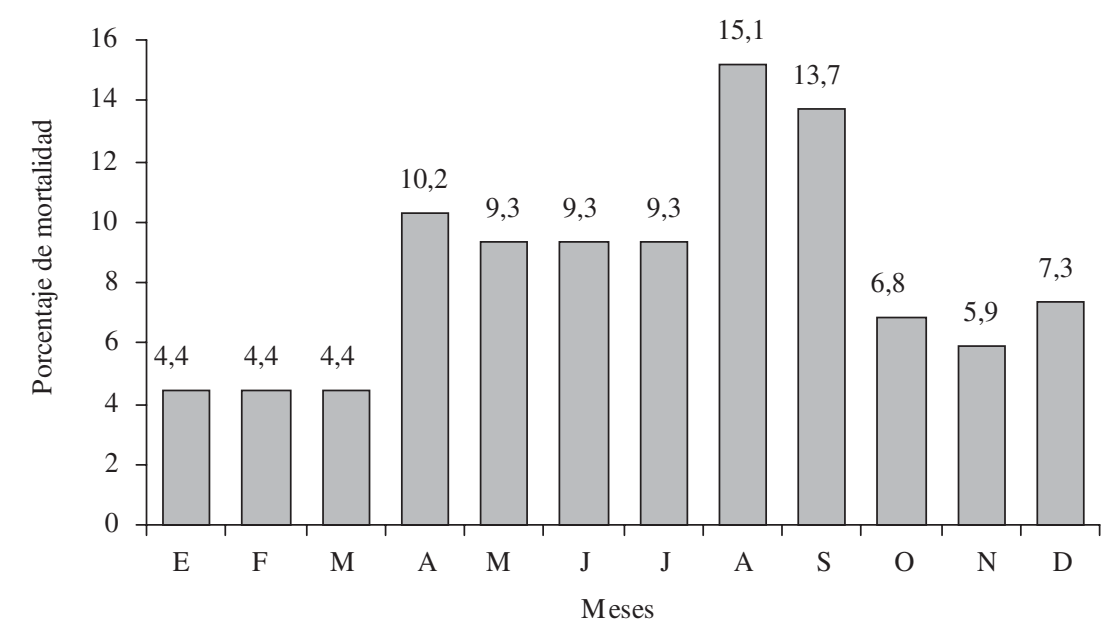

Figura 1. Distribución porcentual de vacas muertas según mes del año en tres rebaños lecheros del sur de Chile (1994-2004).

Proportional distribution of dead cows according to the month of the year in three dairy herds of southern of Chile (1994-2004). 


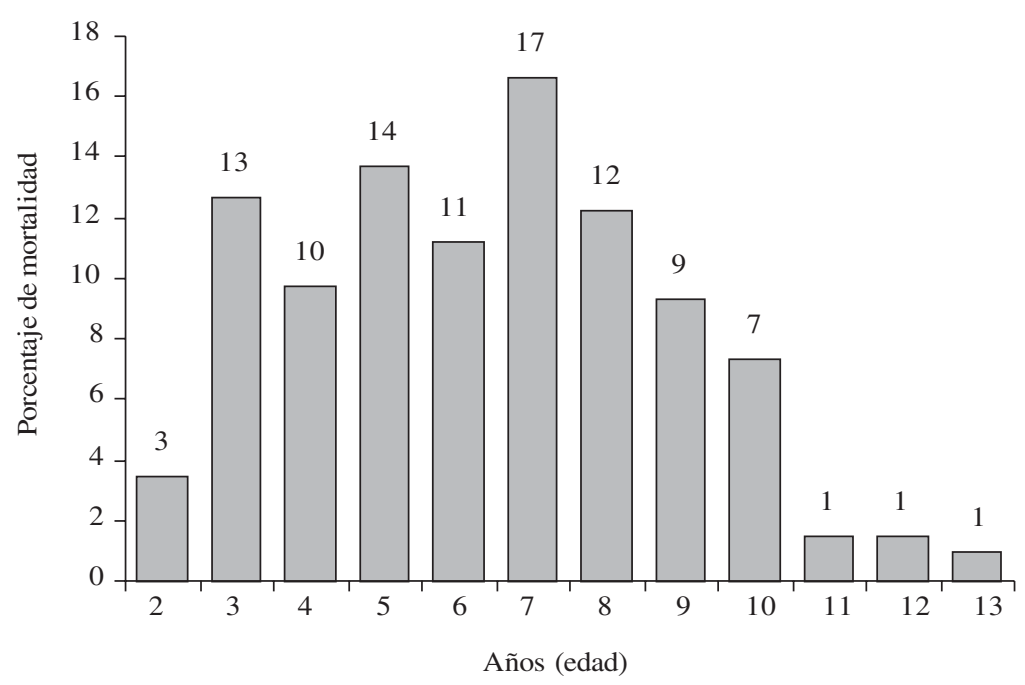

Figura 2. Distribución porcentual de vacas muertas según edad, en tres rebaños lecheros del sur de Chile (1994-2004).

Proportional distribution of dead cows according to age in three dairy herds of southern Chile (1994-2004).

Se calculó la edad promedio de muerte que fue de $6+2$ años (media + DE) considerando las vacas de los tres rebaños. Esto coincide en cierto grado con lo señalado por un estudio realizado en México donde la duración promedio de vida fue de 5,7 + 2,7 años (Márquez y col 2001).

En la distribución de las principales causas de muerte de las vacas dependiendo de la edad, se indica para los trastornos derivados del parto y trastornos digestivos que el mayor número de vacas que murió por estas causas tenían 3 años y que para los trastornos metabólicos/ nutricionales la mayoría de las vacas tenían 8 años (figura 3). Norgaard y col (1999) señalan que muchas enfermedades relacionadas con la producción se desarrollan lentamente, y algunas se mantienen durante mucho tiempo (por ejemplo enfermedades de la pezuña), por lo tanto, los índices de mortalidad por estas enfermedades aumentan con la edad.

Porcentajes de mortalidad según etapa de lactancia de las vacas. La mayoría de las vacas registradas en el estudio murieron durante la primera etapa de la lactancia alcanzando un promedio de $61,8 \%$ (rebaño A $62,7 \%$, rebaño B 58,6\% y rebaño C $64 \%$ ). Esto es similar a lo señalado por un estudio, que indica que el riesgo de muerte es tres veces mayor alrededor del parto que a los 300 días de lactancia (Márquez y col 2001). En el $2^{\circ}$ tercio se obtuvo un promedio de $23,4 \%$ y en el $3^{\text {er }}$ tercio un $14,8 \%$.

Un $62,3 \%$ de las muertes ocurrió durante los primeros 30 días de lactancia; lo que coincide con diversos estudios que señalan una alta proporción de muertes durante los primeros 15-30 días de lactancia con un 45\% (Menzies y col 1995) y un 30-40\% (Thomsen y col 2004). Thomsen y col (2004) indican además que la mortalidad entre vacas viejas ( 3 partos y más) fue aproximadamente el doble que la de vacas jóvenes (1 y 2 partos) durante los 30 primeros días de lactancia.

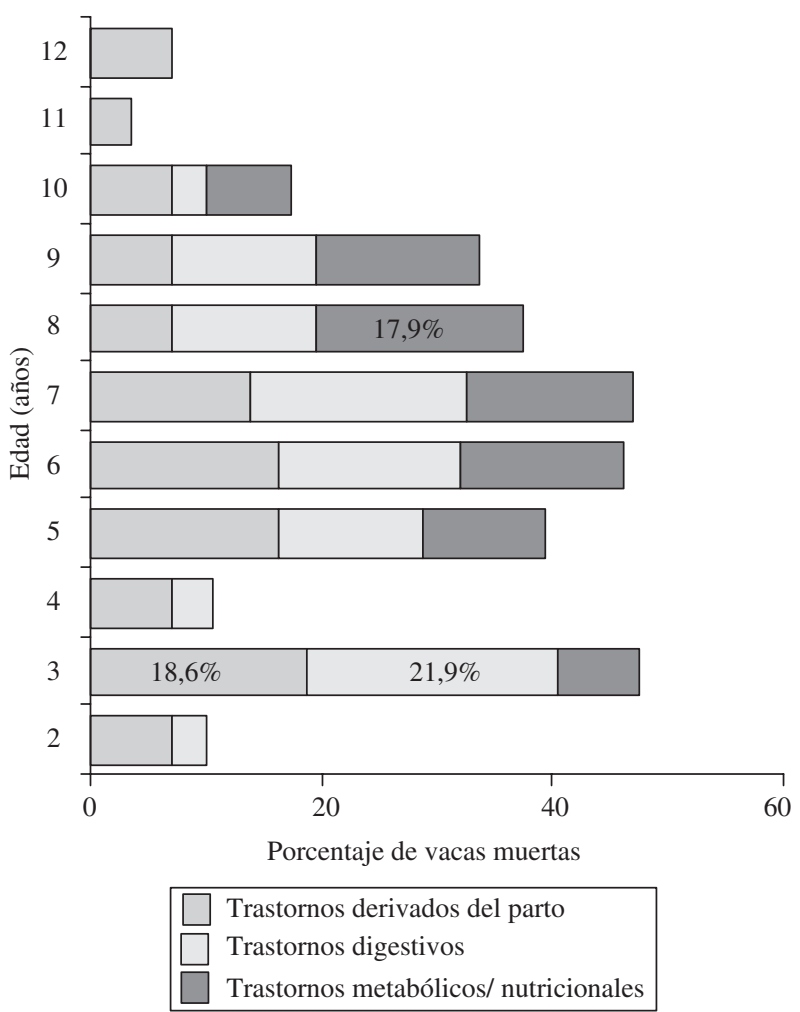

Figura 3. Distribución de las principales causas de muerte de vacas lecheras según edad, en tres rebaños lecheros del sur de Chile (1994 -2004).

Distribution of the main causes of death of dairy cows according to age in three dairy herds of southern Chile (1994-2004). 
En base a los resultados se concluye que la tasa de mortalidad bruta promedio de los tres rebaños es superior a lo reportado por diferentes autores extranjeros, debiendo considerarse las diferencias propias de las condiciones locales de cada estudio.

Debido a que en Chile la información respecto a estudios de mortalidad en vacas es muy escasa, los resultados descritos entregan información que puede servir como base para la toma de decisiones de futuros programas de control y prevención de enfermedades; además de estimular el registro en forma sistemática de las causas muertes de los animales y el manejo adecuado de los cadáveres.

\section{RESUMEN}

El objetivo del estudio fue determinar la tasa de mortalidad bruta promedio anual de las vacas lecheras de tres rebaños de la provincia de Valdivia, Chile, durante el período 1994-2004, además de determinar las causas más frecuentes de muerte. La información se recopiló desde 205 tarjetas de registros individuales de las vacas muertas y de los respectivos inventarios anuales; los datos obtenidos fueron procesados mediante el programa Microsoft Excel. La tasa de mortalidad bruta promedio para el período en estudio fue de un $4,8 \%$ considerando los tres rebaños, mientras que la tasa de mortalidad bruta anual para el rebaño A fue de $6,8 \%$, rebaño B $4,2 \%$ y rebaño C $3,2 \%$. Las causas más frecuentes de muerte, considerando los tres predios en conjunto, fueron los trastornos derivados del parto (21\%), las causas no determinadas $(16,1 \%)$ y los trastornos digestivos $(15,6 \%)$. Los meses del año en donde se presentaron los mayores porcentajes de mortalidad fueron entre abril y septiembre. Con relación a la edad, la mayoría de las vacas en el momento de morir tenía siete años (17\%). La primera etapa de la lactancia fue el período durante el cual murió el mayor porcentaje de las vacas $(61,8 \%)$. Se concluye que existe una alta tasa de mortalidad en los planteles lecheros, siendo las causas de muerte diferentes dependiendo del predio, pero en general se resume que los trastornos derivados del parto, causas no determinadas y los trastornos digestivos son las que prevalecen.

\section{REFERENCIAS}

Costa E F, E Fazzio, R O Traveria, M F Alvarado, G A Mattioli, M M Otero, M Chialva, J R Romero. 2004. Causas de mortalidad y aborto en bovinos. Informe de 1.163 casos entre 1986 y 2001 en la provincia de Buenos Aires. Rev Med Vet 85, 16-22.

Drackley J K. 1999. Biology of dairy cows during the transition period: the final frontier? J Dairy Sci 82, 2259-2273.

Enyedi S. 1991. The degree and causes of cow loss as related to genotypes, keeping systems and herd size. World Rev Anim Prod 26, 29-34.

Esslemont R J, M A Kossaibati. 1997. Culling in 50 dairy herds in England. Vet Rec 140, 36-39.

Faye B, L Pérochon. 1995. La mortalité des vaches laitières dans 1'enquête écopathologique Bretagne. Vet Res 26, 124-131.

Gardner I A, D Hird, W Utterback, C Danaye-Elmi, B Heron, K Christiansen, W Sischo. 1990. Mortality, morbidity, case-fatality, and culling rates for California dairy cattle as evaluated by the National Animal Health Monitoring System, 1986-87. Prev Vet Med 8, 157-170.

Gómez J E. 1992. Causas de mortalidad bovina y su impacto económico en la zona Uraba Antioqueño en el período enero 1991- enero 1992. Actualidad Regional Instituto Colombiano Agropecuario $6,1-5$.

Markusfeld O. 1987. Periparturient traits in seven high dairy herds. Incidence rates, association with parity, and interrelationships among traits. J Dairy Sci 70, 158-165.

Márquez H O, V L M Granados, S D A Arias, C R Espinosa, G F F Sánchez. 2001. Longevidad y factores de riesgo de muerte en vacas lecheras. XXV Congreso Nacional de Buiatría, Veracruz, México, Pp 228-231.

Menzies F D, D G Bryson, T McCallion, D I Matthews. 1995. A study of mortality among suckler and dairy cows in Northern Ireland in 1992. Vet Rec 137, 531-536.

Miles D G, B W Hoffman, K C Rogers, J E Sears. 1998. Diagnosis of digestives deaths. J Anim Sci 76, 320 -322.

Norgaard N H, K M Lind, J F Ageer. 1999. Cointegration analysis used in a study of dairy cow mortality. Prev Vet Med 42, 99-119.

Prasad S, N Ramachandran, S Raju. 2004. Mortality patterns in dairy animals under organized herd management conditions at Karnal, India. Trop Anim Health Pro 36, 645-654.

Stevenson M A, I J Lean. 1998. Descriptive epidemiological study on culling and deaths in eight dairy herds. Aust Vet J 76, 482- 488.

Thomsen P T, A M Kjeldsen, J T Sorensen, H Houe. 2004. Mortality (including euthanasia) among Danish dairy cows (1990-2001). Prev Vet Med 62, 19-33.

Thrusfield M. 1990. Epidemiología veterinaria. Editorial Acribia S.A., Zaragoza, España.

Wayne S, A H Meek, P Willeberg. 1997. Epidemiología veterinaria. Principios y métodos. Editorial Acribia S.A., Zaragoza, España. 